\title{
Monocyte accumulation is an early event in HAM/TSP pathogenesis, while monocyte activation and IFN-regulated gene expression persist in chronic HAM/TSP
}

\author{
Soraya Maria Menezes ${ }^{1}$, Harry Freitag Muhammad', George Soares², Ricardo Khouri, ${ }^{1,2}$, Daniele Decanine ${ }^{2}$, \\ Gilvaneia Silva Santos², Saul Velloso Schnitman², Ramon Kruschewsky33, Giovanni López ${ }^{4}$, Carolina Alvarez “4, \\ Michael Talledo ${ }^{4}$, Eduardo Gotuzzo ${ }^{4,5}$, Bernardo Galvão-Castro ${ }^{3}$, Johan Van Weyenbergh ${ }^{1,2^{*}}$
}

From 17th International Conference on Human Retroviruses: HTLV and Related Viruses

Trois Ilets, Martinique. 18-21 June 2015

Tattermusch et al (2012) identified an IFN-inducible gene signature in whole blood of HAM/TSP patients, with a strong myeloid component, while abortive HTLV1 infection induces monocyte apoptosis (Sze et al. 2013). We previously demonstrated that B cell CD80 expression correlates to disease severity in HAM/TSP (Menezes et al 2014), whereas B cell CD86 is selectively up-regulated by IFN-beta in both HAM/TSP and multiple sclerosis (MS). In this study, we propose a cell type-and gene-specific, rather than a generalized IFN response in HAM/TSP. Using polychromatic flow cytometry, comprehensive phenotyping of monocytes (CD14, CD64, CD80, CD86, CD95/Fas, HLA-DR) was performed in a total of 53 individuals (HAM/TSP patients, asymptomatic HTLV-1infected and uninfected controls), and absolute and relative monocyte counts were obtained from $>600$ HTLV-1-infected individuals with complete clinical follow-up and proviral load. Ex vivo monocyte levels increased in early HAM/TSP $(\mathrm{p}<0.01)$, independent of proviral load, and were significantly correlated to age of onset of HAM/TSP in both Brazilian and Peruvian cohorts. On the other hand, monocyte activation measured by systemic soluble CD14 was significantly increased in chronic $(\mathrm{p}<0.01)$ but not early HAM/TSP, whereas CD95 and CD86 expression in monocytes correlated negatively to disease progression. Interestingly,

\footnotetext{
* Correspondence: johan@bahia.fiocruz.br

1 Rega Institute for Medical Research, Department of Microbiology and Immunology K.U, Leuven, Belgium

Full list of author information is available at the end of the article
}

membrane expression of CD14 is down-regulated and CD95/CD86 up-regulated by IFN-beta in vitro (controls) and in vivo (MS), suggesting IFN-regulated expression of all three monocyte receptors in HAM/TSP. Transcriptomic analysis of whole blood vs. purified monocytes/ B cells confirmed cell-specific expression of CD64/ CD80/CD86 ex vivo, whereas a selective decrease of myeloid/monocyte-specific genes was observed upon in vitro culture of HAM/TSP PBMCs, possibly due to apoptosis of specific monocyte subsets. In conclusion, an increase in soluble CD14, as well as monocyte-specific expression of CD64, CD95 and CD86 differentially reflect disease progression in HAM/TSP, in addition to B-cell specific CD80 expression, arguing for a complex and compartmentalized IFN response.

\section{Authors' details}

${ }^{1}$ Rega Institute for Medical Research, Department of Microbiology and Immunology K.U, Leuven, Belgium. ${ }^{2}$ LIMI-CPqGM, Oswaldo Cruz Foundation (FIOCRUZ), Salvador-Bahia, Brazil. ${ }^{3}$ Bahiana School of Medicine and Public Health, Salvador-Bahia, Brazil. ${ }^{4}$ Instituto de Medicina Tropical Alexander von Humboldt, Universidad Peruana Cayetano Heredia, Lima, Peru.

${ }^{5}$ Departamento de Medicina, Facultad de Medicina Alberto Hurtado, Universidad Peruana Cayetano Heredia, Lima, Peru.

Published: 28 August 2015

$$
\begin{aligned}
& \text { doi:10.1186/1742-4690-12-S1-O25 } \\
& \text { Cite this article as: Menezes et al.: Monocyte accumulation is an early } \\
& \text { event in HAM/TSP pathogenesis, while monocyte activation and IFN- } \\
& \text { regulated gene expression persist in chronic HAM/TSP. Retrovirology } 2015 \\
& \text { 12(Suppl 1):O25. }
\end{aligned}
$$

